\title{
The 3D restricted three-body problem under angular velocity variation
}

\author{
K. E. Papadakis \\ Department of Engineering Sciences, Division of Applied Mathematics and Mechanics, University of Patras, \\ 26504 Patras, Greece \\ e-mail: K.Papadakis@des.upatras.gr
}

Received 3 May 2004 / Accepted 23 June 2004

\begin{abstract}
Nonlinear approximation of periodic motions around the collinear equilibrium points in the case of the restricted three-body problem when the angular velocity of the primaries is not equal to the value of the classical problem (which is unit in the usual units of mass, length and time), is studied. The stability of the equilibrium points and the analytical solutions in their neighborhood constructing series approximations of the periodic orbits in the planar and in the spatial problem, are given. Families which emanate from $L_{1}, L_{2}$ and $L_{3}$ both in the plane and in three dimensions as well as their stability for the Earth-Moon mass distribution, are computed. Special generating plane orbits, the vertical-critical orbits, of the families $a, b$ and $c$ of the problem, are determined and presented. We have also computed series of vertical-critical periodic orbits with the angular velocity as parameter. Three-dimensional families which generate from the bifurcation orbits for $\omega=2$, are given.
\end{abstract}

Key words. celestial mechanics - methods: numerical

\section{Introduction and equations of motion}

In this paper we treat the problem of periodic orbits emanating from the collinear equilibrium points, of the restricted three-body problem for different angular velocities of the rotating frame. We study the existence and evolution of the two and of three-dimensional periodic orbits of the problem at the collinear equilibrium configurations, both analytically and numerically. Periodic orbits of the restricted three-body problem have a well-known significance in Celestial Mechanics as well as in other branches of science. Well known is also the necessity for tedious numerical calculations in their determination as established for most types of periodic motions. However, analytic approximations of such orbits are possible in the case of small motions around equilibrium points and the usefulness of such approximations is not limited by their local validity because they provide appropriate tools for the initiation of calculations for the numerical determination of entire families of large orbits emanating from the corresponding equilibria.

The interest in the restricted three body problem when the angular velocity receives different values, compared to the classical case, has been revived recently. Chermnykh (1987) began the study of the Lyapunov stability of the triangular solutions, Goździewski \& Maciejewski (1998), investigate Lyapunov stability of Lagrangian libration points in detail in the whole range of parameters $\omega, \mu$ of the problem and Perdios \& Ragos (2004), study the non-symmetric asymptotic motion to the collinear equilibrium points and discuss their relation to families of symmetric periodic solutions. All studies, mentioned above, are done in the planar case of the problem. In the present work we study the symmetric periodic motions which emanate from the Lagrangian equilibrium points $L_{1,2,3}$ and develop their second-order analytic approximations. Series approximations up to third-order in $x, y$ and fourth-order terms in $z$, of the spatial periodic solution, are also given. All families which emanate from the inner equilibrium points (initial conditions are obtained by our analysis, around the equilibria), are numerically calculated up to their terminal points, both in the plane and in three dimensions.

Vertical-critical periodic orbits of the classical restricted three-body problem have been determined numerically by Hénon \& Guyot (1974) and Hénon (1973). Here, we study the stability and we calculate the vertical-critical periodic orbits of the families which emanate from the equilibrium points and in numerical form the series of vertical-critical periodic orbits of these families for the Earth-Moon mass distribution for various values of the angular velocity of the primary bodies. Specifically, we compute, for the appropriate range of the angular velocity parameter $\omega$, the vertical-critical orbits of the basic simple families $a, b$ and $c$. The fact that the orbits in the Solar system are almost flat and the simplifications achieved in considering plane and symmetric periodic solutions of the problem justify computing and studying the stability parameters of the vertical-critical periodic orbits.

Three dimensional solutions, from vertical-critical periodic orbits, are determined and presented. In addition, we compare 
our results with those of the classical restricted three-body problem and present similarities and differences.

The present problem concerns the motion of a particle having infinitesimal mass in the orbital plane of a dumb-bell which is rotating with constant angular velocity $\omega$ around its mass center and so generalizes two classical problems of celestial mechanics, i.e. the two fixed centers problem and the restricted three-body problem, This reason gives the possibility for many applications in celestial mechanics and chemistry (for details see Goździewski \& Maciejewski 1998, and references therein).

The equations of motion of the 3D restricted three-body problem when $\omega$ is the angular velocity of the primaries, with the origin resting at the center of mass, in a rotating system of coordinates and using dimensionless variables (Marchal 1990), may be written as,

$\ddot{x}-2 \omega \dot{y}=\frac{\partial \Omega}{\partial x}=\omega^{2} x-\frac{(1-\mu)(x+\mu)}{r_{1}^{3}}-\frac{\mu(x+\mu-1)}{r_{2}^{3}}$,

$\ddot{y}+2 \omega \dot{x}=\frac{\partial \Omega}{\partial y}=\omega^{2} y-\frac{(1-\mu) y}{r_{1}^{3}}-\frac{\mu y}{r_{2}^{3}}$,

$\ddot{z}=\frac{\partial \Omega}{\partial z}=-\frac{(1-\mu) z}{r_{1}^{3}}-\frac{\mu z}{r_{2}^{3}}$,

where dots denote time derivatives and $\Omega$ the gravitational potential in synodic coordinates is given as

$\Omega=\frac{1}{2} \omega^{2}\left(x^{2}+y^{2}\right)+\frac{1-\mu}{r_{1}}+\frac{\mu}{r_{2}}$,

where

$r_{1}^{2}=(x+\mu)^{2}+y^{2}+z^{2}, \quad r_{2}^{2}=(x+\mu-1)^{2}+y^{2}+z^{2}$.

Also, $\mu$ is the mass-ratio of the smaller primary to the total mass of the primaries, $0<\mu<\frac{1}{2}$ (placing the smaller primary to the left, see Szebehely 1967) and the unit of distance is the distance between the primaries.

The energy (Jacobi) integral of this problem, is given by the expression

$C=2 \Omega-\left(\dot{x}^{2}+\dot{y}^{2}+\dot{z}^{2}\right)$,

where $C$ is the Jacobian constant.

\section{Periodic orbits around the collinear equilibrium configuration}

\subsection{Linearized equations of motion}

The restricted three-body problem with angular velocity of primaries $\omega$ has five equilibrium points (as in the classical restricted problem), three collinear $L_{1,2,3}$, for $\omega \in(0, \infty)$ on the $x$-axis ( $L_{3}$ on the negative side of this axis) and two triangular $L_{4,5}$, for $\omega \in(0,2 \sqrt{2})$. In the limiting case $\omega=2 \sqrt{2}$ they coincide with $L_{1}$ (Goździewski \& Maciejewski 1998). From the differential equations of motion (1) we obtain the coordinates $x_{0}, y_{0}$, of the Lagrangian equilibrium points as solutions of the equations:

$\Omega_{x}=x_{0}\left[\omega^{2}-\frac{1-\mu}{r_{10}^{3}}-\frac{\mu}{r_{20}^{3}}\right]-\mu(1-\mu)\left(\frac{1}{r_{10}^{3}}-\frac{1}{r_{20}^{3}}\right)=0$,

$\Omega_{y}=y_{0}\left[\omega^{2}-\frac{1-\mu}{r_{10}^{3}}-\frac{\mu}{r_{20}^{3}}\right]=0$, where $r_{10}$ and $r_{20}$ are the distances given by Eq. (3) at the equilibrium. For the triangular points $L_{4,5}(y \neq 0)$, we obtain the exact coordinates:

$x_{0}=\frac{1-2 \mu}{2}, \quad y_{0}= \pm \sqrt{\omega^{-4 / 3}-\frac{1}{4}}$

(Chermnykh 1987), i.e. their positions are symmetrical with respect to the $x$-axis and their corresponding Jacobi constant is

$C_{L_{4,5}}=3 \omega^{2 / 3}-\mu(1-\mu) \omega^{2}$.

The linearized equations for infinitesimal motions near the collinear equilibrium points are

$\left(\begin{array}{c}\dot{\boldsymbol{x}} \\ \dot{z}\end{array}\right)=\left(\begin{array}{cc}A_{4 \times 4} & 0_{4 \times 2} \\ 0_{2 \times 4} & B_{2 \times 2}\end{array}\right)\left(\begin{array}{l}\boldsymbol{x} \\ \boldsymbol{z}\end{array}\right), \quad \begin{aligned} & \boldsymbol{x}=(x, y, \dot{x}, \dot{y})^{T}, \\ & \boldsymbol{z}=(z, \dot{z})^{T},\end{aligned}$

where $(\boldsymbol{x}, \boldsymbol{z})$ is the state vector of the third particle with respect to the equilibrium points and the time-independent coefficient matrixes $A$ and $B$ are:

$A=\left(\begin{array}{cccc}0 & 0 & 1 & 0 \\ 0 & 0 & 0 & 1 \\ A_{0} & 0 & 0 & 2 \omega \\ 0 & A_{1} & -2 \omega & 0\end{array}\right), \quad B=\left(\begin{array}{cc}0 & 1 \\ -B_{0} & 0\end{array}\right)$,

where

$A_{0}=\omega^{2}+2 A_{2}, \quad A_{1}=\omega^{2}-A_{2}$,

$A_{2}=\frac{(1-\mu)\left|x_{0}+\mu\right|}{\left(x_{0}+\mu\right)^{4}}+\frac{\mu\left|x_{0}+\mu-1\right|}{\left(x_{0}+\mu-1\right)^{4}}$,

$B_{0}=\frac{1-\mu}{\left|x_{0}+\mu\right|^{3}}+\frac{\mu}{\left|x_{0}+\mu-1\right|^{3}}$.

\subsection{Planar periodic orbits}

To study analytically the solutions in the neighborhood of the equilibrium configuration $L_{i}, i=1,2,3$, as we already mentioned, the origin is transferred at $L_{i}$, the equations of motion are linearized and receive for coplanar motion, the expression $\dot{\boldsymbol{x}}=A \boldsymbol{x}$. The characteristic equation of the matrix $A$ is thus

$\lambda^{4}+\left(2 \omega^{2}-A_{2}\right) \lambda^{2}+\omega^{4}+A_{2} \omega^{2}-2 A_{2}^{2}=0$.

and the corresponding eigenvalues are

$\lambda_{1,2,3,4}= \pm \frac{1}{\sqrt{2}} \sqrt{A_{2}-2 \omega^{2} \pm \sqrt{A_{2}} \sqrt{9 A_{2}-8 \omega^{2}}}$.

Hence the collinear Lagrangian points are always unstable (Perdios et al. 2004), except for the case when $\omega=2 \sqrt{2}$ in which the triangular points $L_{4,5}$ coalesce with the inner equilibrium point $L_{1}$. In that case the collinear point is stable (Goździewski et al. 1998).

Using a standard perturbations technique (see, e.g. Papadakis et al. 1988), we can find approximately the periodic orbits which emanate from the equilibrium points $L_{1,2,3}$. A second-order approximation, with respect to a small orbital parameter $\epsilon$, of the periodic solution of the problem, is

$x(t)=x_{0}+\cos \omega_{\mathrm{p}} t \epsilon+\left(c_{11}+c_{12} \cos 2 \omega_{\mathrm{p}} t\right) \epsilon^{2}+O\left(\epsilon^{3}\right)$,

$y(t)=c_{21} \sin \omega_{\mathrm{p}} t \epsilon+c_{22} \sin 2 \omega_{\mathrm{p}} t \epsilon^{2}+O\left(\epsilon^{3}\right)$, 
where $\left(x_{0}, 0\right)$ is given by Eq. (5), the coefficients $c_{i j}, i=1,2$ are constants depending on the mass parameter $\mu$ the equilibrium point distance $x_{0}$ and the quantity $\omega$, i.e., ultimately only on $\mu$ and $\omega$. Two of the four eigenvalues (12) are real (responsible for the instability of the equilibria) and the other two are the pure imaginary:

$\pm i \omega_{\mathrm{p}}$ with $\omega_{\mathrm{p}}=\frac{1}{\sqrt{2}} \sqrt{2 \omega^{2}-A_{2}+\sqrt{A_{2}} \sqrt{9 A_{2}-8 \omega^{2}}}$.

Specifically we have:

$c_{21}=-\frac{\omega^{2}+2 A_{2}+\omega_{\mathrm{p}}^{2}}{2 \omega \omega_{\mathrm{p}}}$,

$c_{11}=\frac{3 A_{3}\left(2-c_{21}^{2}\right)}{4 A_{0}}$,

$c_{22}=\frac{3 A_{3}\left[2 \omega \omega_{\mathrm{p}}\left(2+c_{21}^{2}\right)+c_{21}\left(A_{0}+4 \omega_{\mathrm{p}}^{2}\right)\right]}{2\left[16 \omega^{2} \omega_{\mathrm{p}}^{2}-\left(A_{0}+4 \omega_{\mathrm{p}}^{2}\right)\left(A_{1}+4 \omega_{\mathrm{p}}^{2}\right)\right]}$,

$c_{12}=-\frac{1}{4 \omega \omega_{\mathrm{p}}}\left[\frac{3}{2} A_{3} c_{21}+\left(A_{1}+4 \omega_{\mathrm{p}}^{2}\right) c_{22}\right]$,

where

$A_{3}=\frac{(1-\mu)\left|x_{0}+\mu\right|}{\left(x_{0}+\mu\right)^{5}}+\frac{\mu\left|x_{0}+\mu-1\right|}{\left(x_{0}+\mu-1\right)^{5}}$

and $A_{0}, A_{1}, A_{2}$ are given by Eq. (11). The accuracy of this analytic approximation to the periodic solutions of the complete equations of the restricted three-body problem with angular velocity of the primaries $\omega$ "around" the equilibrium configuration depends, obviously, on the size of the arbitrary orbital parameter $\epsilon$.

In Fig. 1a, we present the characteristic curves (dashed lines indicate positive crossings of the $x$-axis) of the families $a, b$ and $c$ (same notation as Hénon 1969), which emanate from $L_{2}$, $L_{3}$ and $L_{1}$ respectively, in the $(x, C)$ plane, for the Earth-Moon mass distribution $\mu=0.01213$ and for $\omega=2$. All the families consist of simple (i.e. orbits which intersect the $x$-axis only twice per period) symmetric, with respect to $x$-axis, periodic orbits and they illustrated in Figs. $1 \mathrm{~b}, 1 \mathrm{c}$ and $1 \mathrm{~d}$. The families $b$ and $c$ are "terminated" by coalescing with the Earth and the family $a$ is reaching the Moon. The periodic orbits of the families $b$ and $a$ are unstable while the family $c$ has a short arc of stable periodic orbits (it is presented with thick line in Fig. 1a). Position of the Earth $\left(m_{1}\right)$ and Moon $\left(m_{2}\right)$ is denoted by dotted lines. As we have mentioned, the energy integral of the problem is given by Eq. (4) and therefore the curves of zero velocity of the problem are defined by $2 \Omega-C=0$, while the regions of possible motions of the third particle are defined by the inequality $2 \Omega-C>0$. In Fig. 1a the forbidden regions are shaded.

\subsection{Three-dimensional periodic orbits}

Following the procedure of the previous subsection, we study analytically the three-dimensional solutions around the equilibrium configurations $L_{1}, L_{2}$ and $L_{3}$. The characteristic equation of matrix $B$ of Eq. (9) has two pure imaginary roots,

$\lambda_{1,2}= \pm i \omega_{z}, \quad \omega_{z}=\sqrt{B_{0}}$
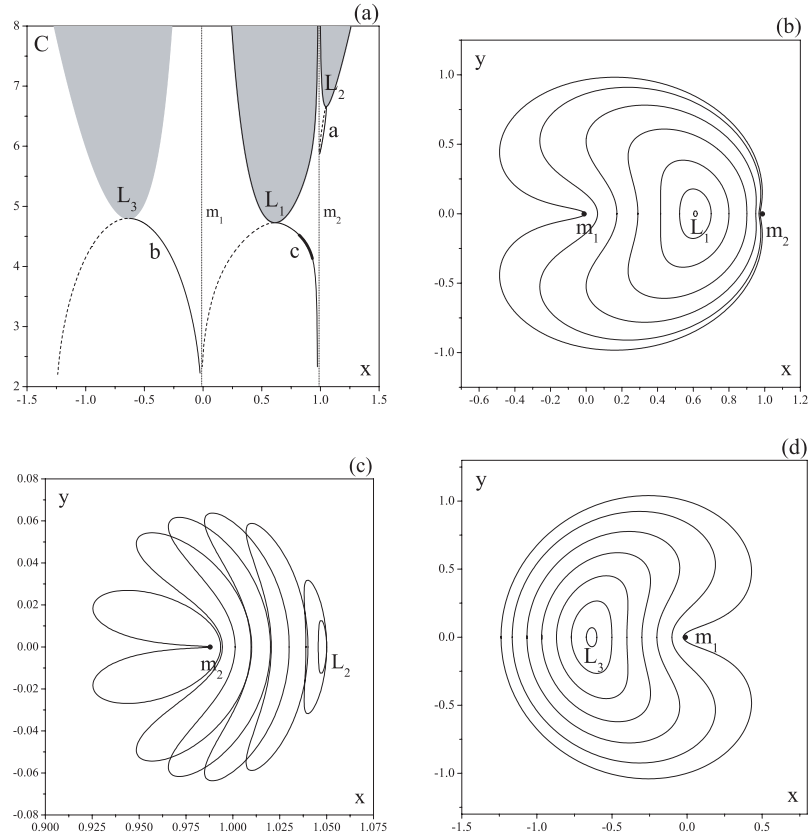

Fig. 1. a) Characteristic curves of the families which emanate from the collinear Lagrangian points for Earth-Moon mass distribution $(\mu=$ 0.01213 ) and $\omega=2$. No motion is possible in the shaded areas (see text). b), c), d) Periodic orbits of these families.

and therefore no instability is caused. The quantity $B_{0}$, defined by relation (11), is positive throughout the range of $\mu$ and $\omega$. Using a perturbations technique and the transformation of the time (see, e.g. Zagouras \& Markellos 1985),

$t=\tau\left(1+a_{2} \epsilon^{2}+O\left(\epsilon^{4}\right)\right)$

we can determine a series approximations up to third order in $x$ and $y$ and fourth order terms in $z$ :

$x(\tau)=x_{0}+\left(z_{11}+z_{12} \cos 2 \omega_{z} \tau\right) \epsilon^{2}+O\left(\epsilon^{4}\right)$,

$y(\tau)=z_{21} \sin 2 \omega_{z} \tau \epsilon^{2}+O\left(\epsilon^{4}\right)$,

$z(\tau)=z_{31} \sin \omega_{z} \tau \epsilon+z_{32} \sin 3 \omega_{z} \tau \epsilon^{3}+O\left(\epsilon^{5}\right)$.

The coefficient $a_{2}$ of the time transformation and the coefficients $z_{i j}$ are:

$$
\begin{aligned}
z_{11}= & -\frac{3 B_{1}}{4 \omega_{z}^{2}\left(\omega^{2}+2 \omega_{z}^{2}\right)}, \\
z_{12}= & \frac{3 B_{1}\left(\omega^{2}+3 \omega_{z}^{2}\right)}{4 \omega_{z}^{2}\left(\omega^{4}-7 \omega^{2} \omega_{z}^{2}+18 \omega_{z}^{4}\right)}, \\
z_{21}= & \frac{3 B_{1} \omega}{-\omega^{4} \omega_{z}+7 \omega^{2} \omega_{z}^{3}-18 \omega_{z}^{5}}, \\
z_{32}= & \frac{3}{8 \omega_{z}^{3}\left(A_{2}-9 \omega_{z}^{2}\right)}\left[\mu B_{2}\left(4 z_{12} \omega_{z}^{2}\left(x_{0}+\mu-1\right)-1\right)\right. \\
a_{2}= & \frac{3}{16 \omega_{z}^{2} A_{2}}\left[4 \omega_{z}^{2} \mu B_{2}\left(\frac{3}{4 x_{0}^{2}+\mu \mid}+\left(x_{z}+\mu-1\right)\left(2 z_{11}-z_{12}\right)\right)\right. \\
& \quad+\frac{\left|x_{0}+\mu\right|}{\left(x_{0}+\mu\right)^{6}}\left(3+4 x_{0} \omega_{12}^{2}\left(2 \omega_{z}^{2}\right)\right],
\end{aligned}
$$


(a)
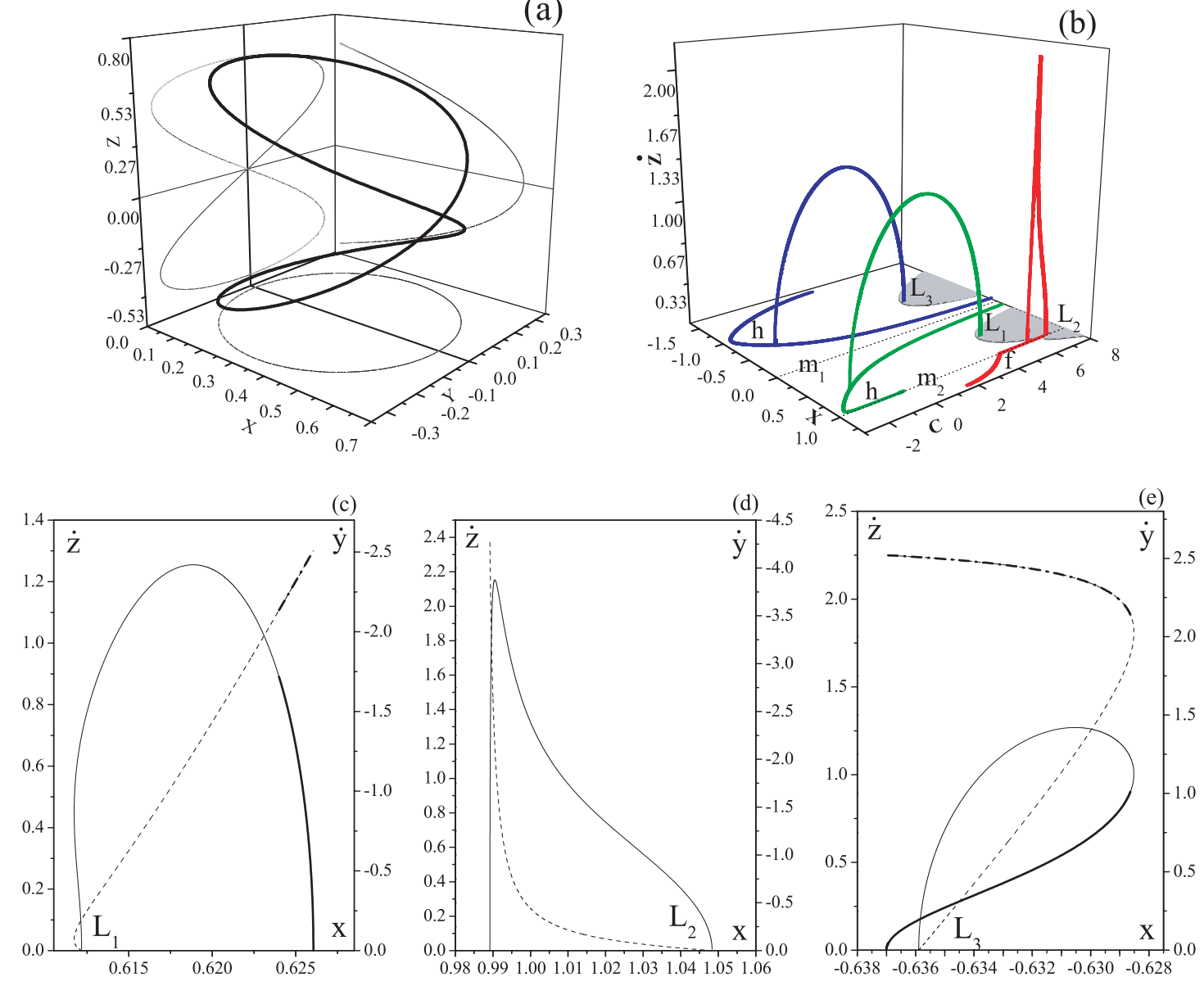

Fig. 2. a) A member of the family of periodic orbits emanating from the equilibrium point $L_{1}$ for $\mu=0.01213$ and $\omega=2$. The projections of the periodic orbit are also shown. b) The evolution of the 3D families which emanate from the equilibrium points. c), d), e) The characteristic curves of the families, in the $(x, \dot{y})$ plane (dashed line) and in the $(x, \dot{z})$ one. In c) and in e) frames the heavy lines correspond to stable periodic orbits.

where

$$
\begin{aligned}
& B_{1}=\frac{1-\mu}{\left|x_{0}+\mu\right|\left(x_{0}+\mu\right)^{3}}+\frac{\mu}{\left|x_{0}+\mu-1\right|\left(x_{0}+\mu-1\right)^{3}} \\
& B_{2}=-\frac{\left|x_{0}+\mu\right|}{\left(x_{0}+\mu\right)^{6}}+\frac{\left|x_{0}+\mu-1\right|}{\left(x_{0}+\mu-1\right)^{6}}
\end{aligned}
$$

and $A_{2}$ is given by Eq. (11).

From the relations (19) we obtain the initial conditions for the numerical determination of the families of the symmetric periodic orbits which emanate from the collinear equilibrium points.

Family from $L_{1}$ : a member of the three-dimensional family of symmetric periodic orbits emanating from the equilibrium point $L_{1}$, for $\omega=2$ and for the Earth-Moon mass distribution $\mu=0.01213$, is shown in Fig. 2a. The orthographic projections in the $(x, y),(x, z)$ and $(y, z)$ planes are illustrated too. The initial conditions of this periodic orbit are: $x=0.61859194$, $\dot{y}=-1.19796628$ and $\dot{z}=1.25403282$. The characteristic curves of this family in the $(x, \dot{y})$ and $(x, \dot{z})$ planes are also given (Fig. 2c). This family begins with unstable periodic orbits but it terminates, on the plane again, with stable ones (thick lines in Fig. 2c). The terminating orbit is also investigated and found to be the vertical-critical orbit of the family $h$ with $x_{0}=0.626056$ and $C=-1.569687$. Family $h$ (we use the same notation as Hénon's paper 1965) consists of retrograde symmetric periodic orbits around the larger primary (Earth).

Family from $L_{2}$ : the family which emanates from $L_{2}$, consists of, entirely, unstable periodic orbits and its characteristic curves are illustrated in Fig. 2d. This family ends on the plane at the vertical-critical periodic orbit of family $f$ with $x_{0}=$ 0.989191 and $C=5.864309$. Family $f$ consists of retrograde symmetric periodic orbits around the smaller primary (Moon).

Family from $L_{3}$ : from the third collinear equilibrium point emanates a family which has unstable members and terminates on the plane again with stable ones (Fig. 2e). The terminating periodic orbit is the vertical-critical orbit of family $h$ with $x_{0}=$ -0.637002 and $C=-1.552474$.

In Fig. $2 b$ it is clearly shown the beginning, the evolution, and the termination of these three-dimensional families. Two of them (from $L_{1}$ and $L_{3}$ ) end on the same planar family $h$, while the third one (from $L_{2}$ ) ends at the planar family $f$. All the families start from $\dot{z}=0$ and after attaining a maximum in $\dot{z}$ they descend back to the plane. All orbits have in space the shape of a figure-of-eight. The periodic orbits of the families which emanate from the equilibrium points $L_{i}, i=1,2,3$ are symmetrical with respect to the $x$-axis and the $(x, y),(x, z)$ planes. 

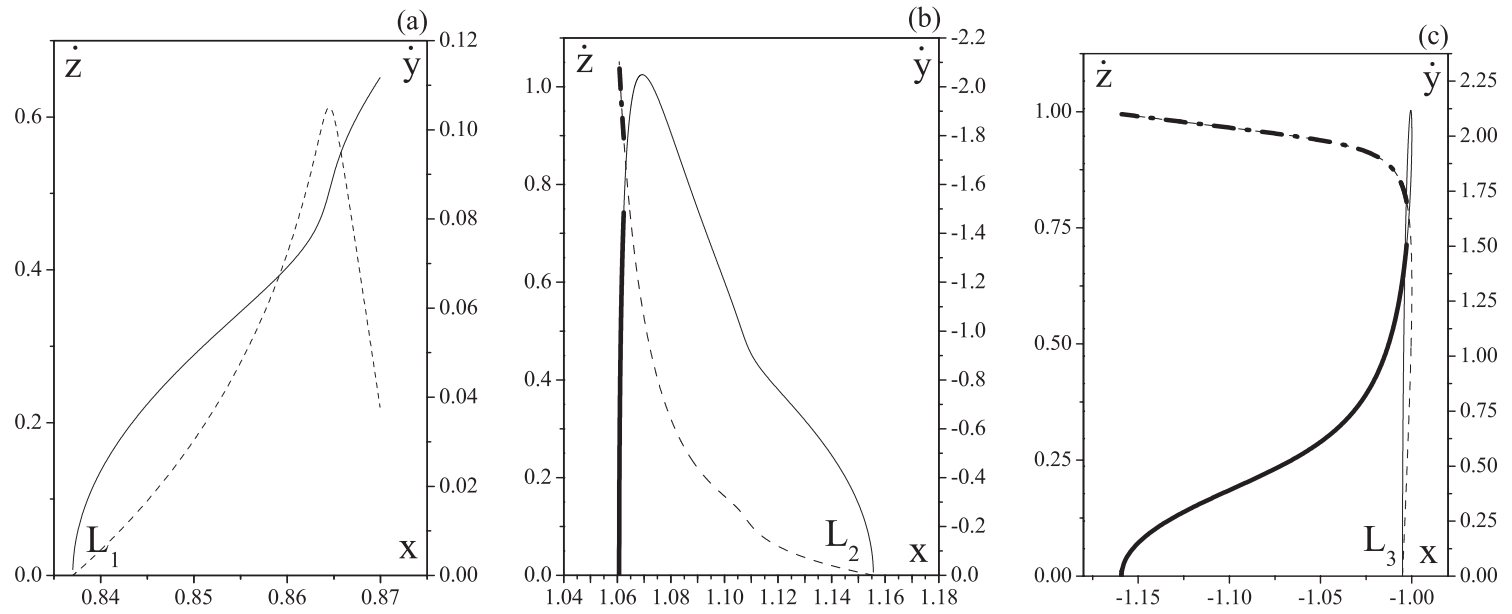

Fig. 3. The corresponding characteristic curves of the three families which emanate from the collinear equilibrium points, in the $(x, \dot{y})$ plane (dashed line) and in the $(x, \dot{z})$ one, in the classical case (i.e. $\omega=1)$, for the Earth-Moon mass distribution. Heavy lines correspond to stable periodic orbits.

The left part of the characteristic curve of the family $h$ is with positive crossing with $x$ axis while the right one is with negative crossing with the same axis (Fig. 2b).

For comparison reasons we have calculated the families which emanate from equilibrium configurations in the classical case, i.e. when the angular velocity is $\omega=1$, for the same mass ratio of the primaries. The results are presented in Fig. 3. The situation is not the similar. The family from $L_{1}$ (Fig. 3a) is entirely unstable and does not return back to the plane but continues with other parts corresponding to higher multiplicity family arc, which is not considered in this paper. We remind here that in all figures, only those families characteristics presented correspond to simple periodic orbits. The other two families (Figs. 3b, 3c) are similar to the ones computed for $\omega=2$, but now the family from $L_{2}$ (Fig. 3b) has a stable part of its characteristic (shown with heavy line).

\section{Vertical-critical periodic orbits}

\subsection{Bifurcations of planar to $3 D$ periodic orbits}

In this section we present the stability of the basic families $a, b$, and $c$ of the restricted problem under angular velocity variation. The horizontal isoenergetic stability of the orbits is computed on the basis of the stability parameter $a_{\mathrm{h}}$ as defined by Hénon (1965). Special generating planar orbits, i.e. the vertical-critical orbits of the problem, are determined for various values of the angular velocity parameter $\omega$ and presented here. Each verticalcritical orbit corresponds to a bifurcation of the family of planar periodic orbits with a family of three dimensional periodic orbits. A periodic orbit is a vertical-critical periodic orbit of unit absolute vertical stability parameter $\left|a_{\mathrm{v}}\right|$ (for details see Hénon 1973). The same orbit is vertically stable for $\left|a_{\mathrm{v}}\right|<1$. In general, an orbit will be three dimensionally stable if and only if it is stable in the plane $\left(\left|a_{\mathrm{h}}\right|<1\right)$ as well as in the third dimension $\left(\left|a_{\mathrm{v}}\right|<1\right)$. The vertical-critical orbits are necessary in order to specify the vertically stable segments of the families of planar periodic orbits, and they are also the starting points of the families of the simplest possible three dimensional periodic orbits, namely the simply and doubly periodic ones. By determining the stability of these orbits we can predict the existence of stable three dimensional periodic orbits, at least for low inclination. So, if a vertical-critical periodic orbit is unstable (therefore horizontally unstable), then for low inclination the generated three dimensional periodic orbit will be unstable too, because of the continuity of the stability parameters. If, on the other hand, a vertical-critical periodic orbit is stable (therefore horizontally stable) then the generated three dimensional periodic orbit may well be stable. For these reasons, the horizontal isoenergetic stability of these vertical-critical periodic orbits were also computed. In Fig. 4 the horizontal and the vertical stability curves of the families $c$ (Fig. 4a), $a$ (Fig. 4b) and $b$ (Fig. 4c), are presented. We have three vertical-critical periodic orbits, denoted by $c i v, i=1, \ldots, 3$ for the Family which emanates from $L_{1}$, and one vertical-critical periodic orbit for each of the other two families, namely $a 1 v$ and $b 1 v$, which emanate from $L_{2}$ and $L_{3}$. Only the family $c$ has an arc of his characteristic curve which consists of stable periodic orbits. Families $a$ and $b$ are entirely horizontally unstable because all the periodic orbits of these families have the stability parameter $\left|a_{\mathrm{h}}\right|<1$. In the next section we will see that this situation is not the same as in the classical restricted three-body problem.

In Table 2 we list the initial conditions of the verticalcritical periodic orbits of the basic families $a, b$ and $c$ of the problem, for $\mu=0.01213$. For each orbit listed in this table, $x_{1}$ denotes the position of the particle on the $x$-axis at $t=T / 2$ and $C$ is the Jacobi constant. There are four types of vertical bifurcations corresponding to the four (essentially three) symmetry types of 3D orbit involved (Hénon 1973; Markellos et al. 1981). Particularly, we have the vertical stability parameters $a_{\mathrm{v}}=u_{33}(T), b_{\mathrm{v}}=u_{36}(T) c_{\mathrm{v}}=u_{63}(T)$ and $d_{\mathrm{v}}=u_{66}(T)$, where $T$ is the full period of the periodic orbit, and they describe the vertical stability properties of it. If the quantity $A_{\mathrm{v}}=$ $v_{33}(T / 2)=0$, then the type of the $3 \mathrm{D}$ orbit is doubly symmetric, if $B_{\mathrm{v}}=v_{36}(T / 2)=0$, it is axisymmetric, if $C_{\mathrm{v}}=v_{63}(T / 2)=0$, it is plane-symmetric and if $D_{\mathrm{v}}=v_{66}(T / 2)=0$, it is doubly 

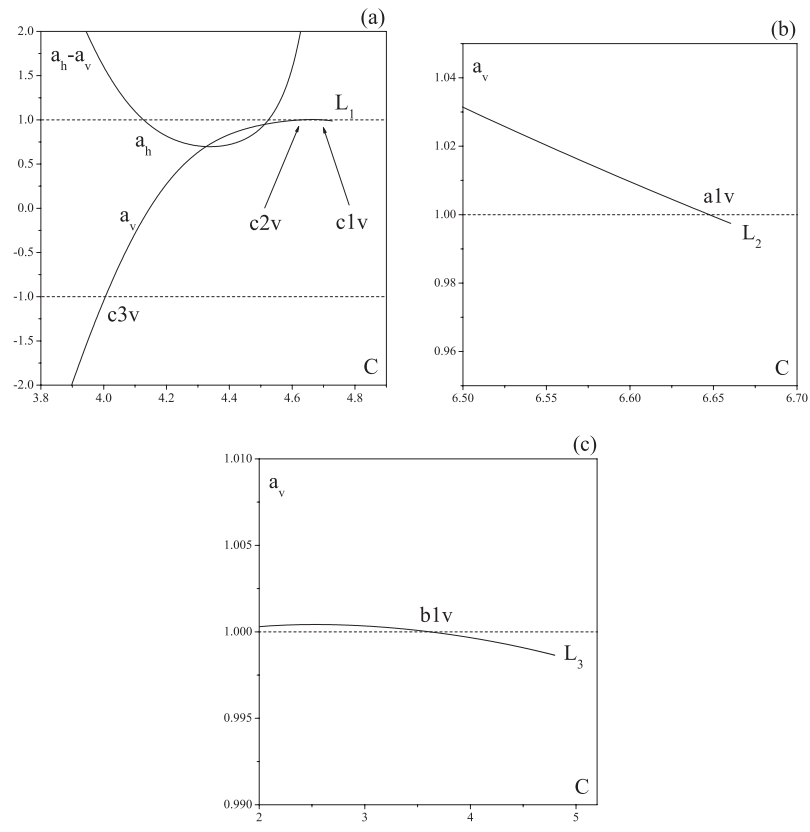

Fig. 4. Vertical stability curves of the families $a, b$ and $c$ which emanate from equilibrium points $L_{2}, L_{3}$ and $L_{1}$ respectively, for EarthMoon mass distribution and for $\omega=2$. Only family $c$ has horizontally stable arc (first frame).

Table 1. Vertical bifurcation conditions.

\begin{tabular}{ll}
\hline \hline Conditions & Type of 3D orbit \\
\hline$a_{\mathrm{v}}=-1, c_{\mathrm{v}}=0\left(A_{\mathrm{v}}=0\right)$ & Doubly symmetric \\
$a_{\mathrm{v}}=1, b_{\mathrm{v}}=0\left(B_{\mathrm{v}}=0\right)$ & Axisymmetric \\
$a_{\mathrm{v}}=1, c_{\mathrm{v}}=0\left(C_{\mathrm{v}}=0\right)$ & Plane symmetric \\
$a_{\mathrm{v}}=-1, b_{\mathrm{v}}=0\left(D_{\mathrm{v}}=0\right)$ & Doubly symmetric \\
\hline
\end{tabular}

symmetric (for more details see Hénon 1973). Conclusively, the bifurcation conditions are:

The bifurcating family to 3D orbits consists of simple periodic (one revolution) orbits if $a_{\mathrm{v}}=1$ and of double periodic (two revolutions) orbits if $a_{\mathrm{v}}=-1$.

The second column of the Table 2 is the angular velocity $\omega$ of the primaries and the last column is the quantity that is zero at the period half and indicates the type of the bifurcating 3D family of periodic orbits.

We can obtain a one-parameter set of vertical-critical periodic orbits by varying the angular velocity parameter $\omega$. If the parameter is fixed the vertical-critical orbits are isolated. In the present case, we computed monoparametric sets of verticalcritical orbits for values of $\omega$ varying in the range [0,3]. Each such set, we call, a series of vertical-critical orbits. In Fig. 5 the characteristic curves of the series of vertical-critical periodic orbits of families $c$ (Fig. 5a) and $a, b$ (Fig. 5b) for Earth-Moon mass distribution, are presented. The initial values $x_{0}$ are shown as full lines and the first intersections $x_{1}$ with the $x$-axis are shown as dashed lines. The two dotted lines in the left frame are the positions of the primary bodies $m_{1}$ and $m_{2}$. The notation of each of the vertical-critical periodic orbit is given. The vertical-critical periodic orbits $c 1 v$ exist for the range $\omega \in[0,2 \sqrt{2}]$, i.e. up to the critical value of $\omega=2 \sqrt{2}$, at which the triangular equilibrium points $L_{4}$ and $L_{5}$ coincide with the inner Lagrangian point $L_{1}$. The vertical-critical periodic orbits $c 2 v$ exist for the range $\omega \in[0.633,2 \sqrt{2}]$. We note that for $\omega=0.633$ the third particle is led to collision with the Moon. The third vertical-critical periodic orbits $c 3 v$ of Family $c$, exist for the range $\omega \in[0.785,2.53]$ and is terminated (in both sides) by collision solutions with the primaries. Consequently, family $c$ has not vertical-critical periodic orbit after the critical value $\omega=2 \sqrt{2}$, for the mass ratio under consideration. All vertical-critical periodic orbits of the series $c 1 v$ computed, are horizontally unstable, while $c 2 v$ and $c 3 v$ have stable and unstable vertical-critical orbits.

Also, in Fig. 5a we present the series of vertical-critical periodic orbits $a 1 v$ and $b 1 v$, which exist in the entire interval considered for the parameter $\omega$. All computed vertical-critical periodic orbits of the series $a 1 v$ and $b 1 v$, are unstable since their horizontal stability parameter $a_{\mathrm{h}}$ satisfies the condition $\left|a_{\mathrm{h}}\right|>1$. Series $a 1 v$ has strongly unstable vertical-critical periodic orbits as $\omega$ increases.

For a global outlook of vertical-critical periodic solutions of the families $a, b$ and $c$, we present now their evolution, as the angular velocity of the primaries varies. We compute the families which emanate from equilibrium points, in the entire range of parameter $\omega$ and for the Earth-Moon mass ratio. For each family we calculate the horizontal and the vertical stability of their periodic orbits and we present the corresponding stability curves. So, in Figs. 6a, $6 \mathrm{~b}$ and $6 \mathrm{c}$ the characteristic curves of the families $c, a$ and $b$ for various values of the angular velocity parameter $\omega$, are presented. The corresponding horizontal and the vertical stability curves of these families follow (Figs. 6d6f). The dotted lines represent the position of the primary bodies $m_{1}$ and $m_{2}$ and the small circle disk denotes the position of the equilibrium point. In Fig. 6a we present the evolution of the family $c$, which emanates from $L_{1}$, for various values of $\omega$. All the families terminate with collision (case) with the Moon (for $\omega=0.5,1,1.5$ ) or with the Earth (for $\omega=2,2.5,2 \sqrt{2}$ ). The initial values of $x$ (negative crossing with $x$-axis) are shown as full lines and the first intersections with the $x$-axis are shown as dashed lines. In the double Fig. 6d we present the corresponding stability curves of these families. We note that in the classical restricted three body problem $(\omega=1)$ the family $c$ is entirely horizontally unstable, but now, when the angular velocity increases, there are periodic solutions which are stable. In the critical value of the parameter $\omega=2 \sqrt{2}$, the family $c$ is stable. Thus the family $c$ has one vertical-critical periodic solution when $\omega=0.5,2 \sqrt{2}$ and three vertical-critical ones for the rest of the values of $\omega$ under consideration.

In Fig. $6 \mathrm{~b}$ we present the evolution of the family $a$ as the angular velocity of the primaries varies, and in the fifth frame we illustrate the corresponding stability curves of these families. All the families terminate with collision cases with the Moon and are entirely horizontally unstable for any value of the parameter $\omega$. In these families we see that in the classical restricted three-body case we have five vertical-critical periodic solutions, but when the parameter $\omega=1.1$ the vertical-critical 
Table 2. Vertical critical periodic orbits of the families $a, b$ and $c$ for Earth-Moon mass distribution $\mu=0.01213$.

\begin{tabular}{|c|c|c|c|c|c|c|}
\hline Name & $\omega$ & $x_{0}$ & $x_{1}$ & $T / 2$ & $C$ & Type \\
\hline \multicolumn{7}{|c|}{ Family $a$} \\
\hline \multirow[t]{5}{*}{$a 1 v$} & 2.5 & 1.03254622 & 1.03557577 & 0.28336388 & 9.08459956 & $v_{63}-C_{\mathrm{v}}$ \\
\hline & 2.0 & 1.04479728 & 1.05020072 & 0.41468172 & 6.64741023 & \\
\hline & 1.5 & 1.06707736 & 1.07992954 & 0.70698545 & 4.68098509 & \\
\hline & 1.0 & 1.12033300 & 1.18080080 & 1.70766779 & 3.15198077 & \\
\hline & 0.5 & 1.39062984 & 1.83815614 & 5.87992791 & 1.88549179 & \\
\hline \multicolumn{7}{|c|}{ Family $b$} \\
\hline \multirow[t]{5}{*}{$b 1 v$} & 2.5 & -0.96093949 & -0.13152480 & 1.23857575 & 4.12883849 & $v_{63}-C_{\mathrm{v}}$ \\
\hline & 2.0 & -1.10736825 & -0.15913015 & 1.55170278 & 3.61497470 & \\
\hline & 1.5 & -1.32484893 & -0.20790741 & 2.07403089 & 3.05872518 & \\
\hline & 1.0 & -1.69676477 & -0.30954860 & 3.11961094 & 2.42351413 & \\
\hline & 0.5 & -2.57582799 & -0.60470992 & 6.25914574 & 1.61362704 & \\
\hline \multicolumn{7}{|c|}{ Family $c$} \\
\hline \multirow[t]{5}{*}{$c 1 v$} & 2.5 & 0.46611541 & 0.59281418 & 1.24501083 & 5.42614478 & $v_{63}-C_{\mathrm{v}}$ \\
\hline & 2.0 & 0.53386915 & 0.68982185 & 1.50402416 & 4.69565752 & \\
\hline & 1.5 & 0.66918153 & 0.78276310 & 1.73922367 & 3.93316244 & \\
\hline & 1.0 & 0.82349738 & 0.85489457 & 1.37158941 & 3.17417618 & \\
\hline & 0.5 & 0.87793339 & 0.88104043 & 0.95236513 & 2.63057241 & \\
\hline \multirow[t]{4}{*}{$c 2 v$} & 2.5 & 0.43040006 & 0.62834419 & 1.25206907 & 5.38857719 & $v_{36}-B_{\mathrm{v}}$ \\
\hline & 2.0 & 0.46931658 & 0.75208823 & 1.54366769 & 4.62963684 & \\
\hline & 1.5 & 0.59459791 & 0.84822833 & 1.92649592 & 3.87103298 & \\
\hline & 1.0 & 0.78170513 & 0.93065808 & 1.97504619 & 3.02139109 & \\
\hline \multirow[t]{4}{*}{$c 3 v$} & 2.5 & 0.01987054 & 0.95714754 & 1.28644158 & 3.28357247 & $v_{66}-D_{\mathrm{v}}$ \\
\hline & 2.0 & 0.19011684 & 0.94518341 & 1.65149397 & 4.00529684 & \\
\hline & 1.5 & 0.44059070 & 0.92968118 & 2.22301844 & 3.72554831 & \\
\hline & 1.0 & 0.71300105 & 0.96011504 & 2.80912492 & 2.94935630 & \\
\hline
\end{tabular}

orbits decrease to three, for $\omega=1.5$ they become two and then there is only one vertical-critical solution. We have the same number of vertical-critical orbits, as in the classical case $(\omega=1)$, when the angular velocity parameter decreases from this value.

In Figs. 6c and 6f (double) we show the characteristic curves of the family $b$, which emanates from $L_{3}$, and their corresponding stability curves, for various values of the parameter $\omega$. In Fig. $6 f$ a magnification area around the line $a_{\mathrm{v}}=1$, is given. All the families $b$ are terminate with collision cases with the Earth and for $\omega>1.5$ they are horizontally unstable. In the classical case $(\omega=1)$, as well as for the cases $\omega=0.5,1.5$, the families have two vertical-critical periodic solutions, while for $\omega=2$ the family has one vertical-critical orbit.

\subsection{Three-dimensional solutions from vertical-critical periodic orbits}

The significance of the vertical-critical orbits as starting points for the determination of the basic families of three-dimensional periodic orbits is evident. In Sect. 2.3, using the approximate
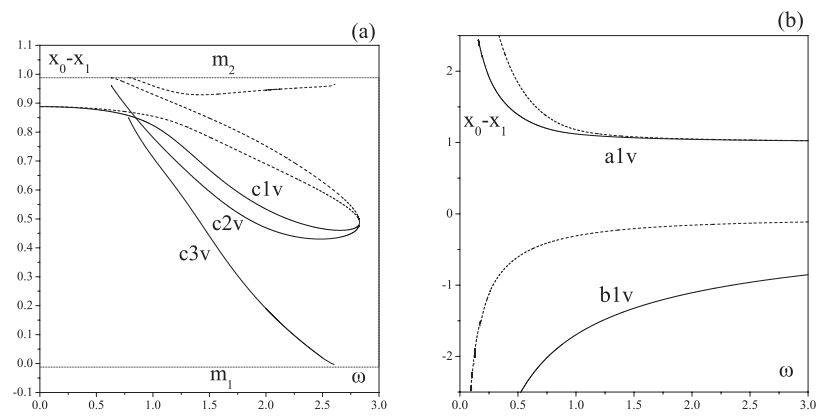

Fig. 5. Characteristic curves of series of vertical-critical periodic orbits of families $a, b$ and $c$ for varying $\omega$. The initial values $x_{0}$ are shown as full lines and the first intersections $x_{1}$ with the $x$-axis are shown as dashed lines. The dotted lines represent the position of the primary bodies $m_{1}$ and $m_{2}$.

solution (19), we calculated the three-dimensional families which emanate from the collinear equilibrium points. In the present subsection, using the planar vertical-critical periodic orbits, already calculated from the previous subsection, we compute the three-dimensional periodic orbits bifurcating 
(a)
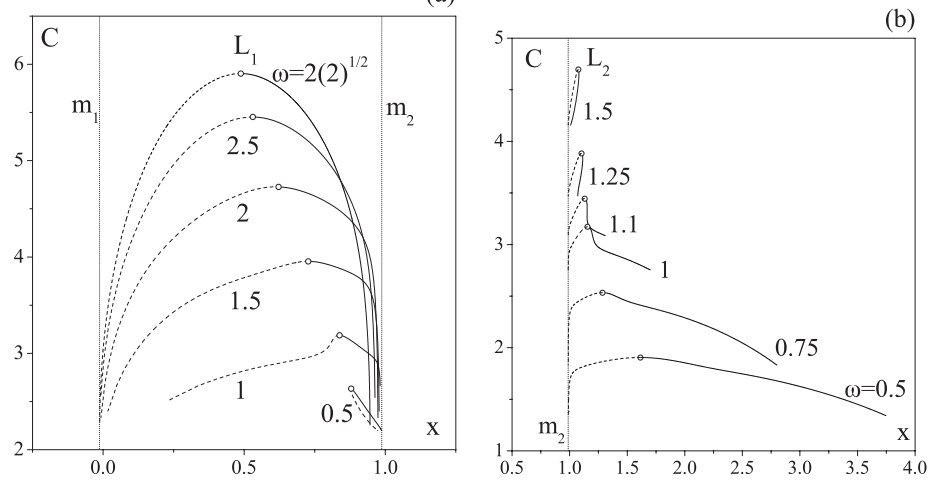

(b)

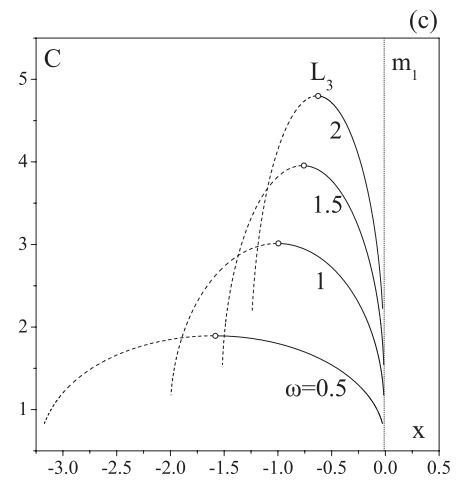

(d)
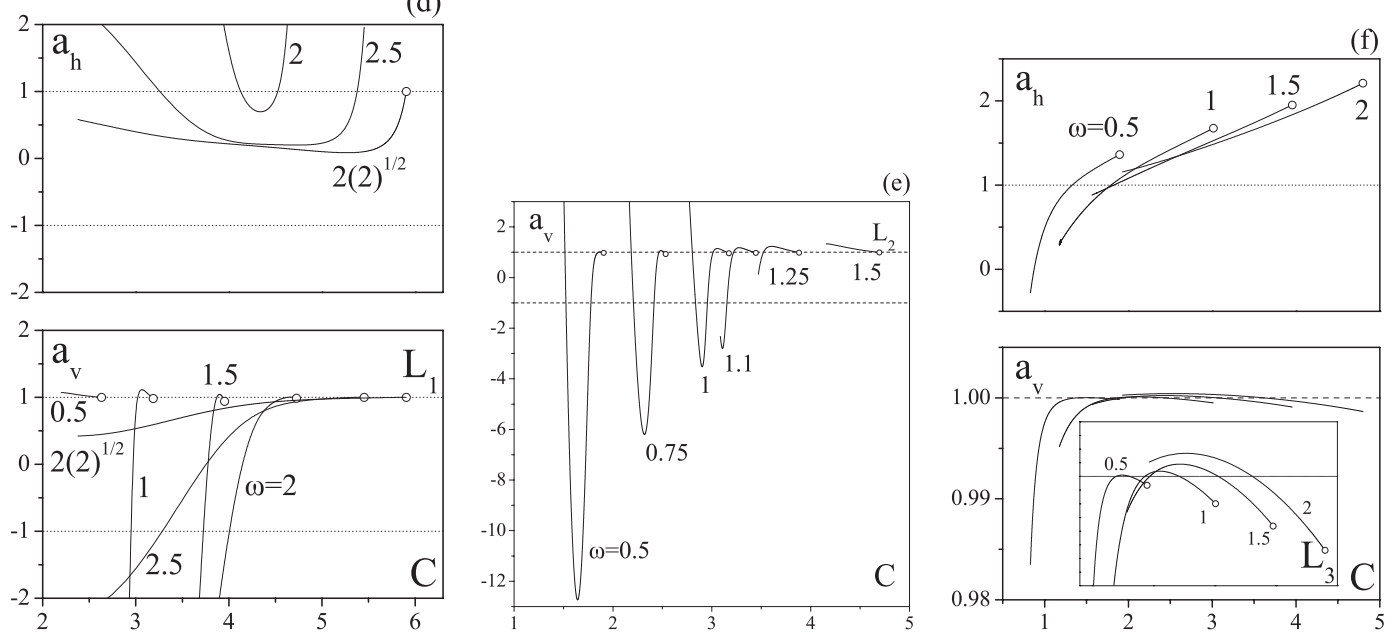

Fig. 6. a), b), c) Characteristic curves of the families $c, a$ and $b$ which emanate from equilibrium $L_{1}, L_{2}$ and $L_{3}$ respectively, for Earth-Moon mass distribution and for various values of the angular velocity $\omega$. The initial values $x$ (negative crossing with $x$-axis) are shown as full lines and the first intersections with the $x$-axis are shown as dashed lines. The dotted lines represent the position of the primary bodies $m_{1}$ or $m_{2}$ and the small circle the position of the equilibrium point. d), e), f) Horizontal and vertical stability curves of these families.

from these planar vertical-critical orbits, for $\omega=2$ and $\mu=$ 0.01213 . The 3D families of these periodic orbits are denoted by the name of the corresponding vertical-critical periodic orbit adding the letter $\mathrm{f}$ in the beginning. So, $f a 1 v$ is the three-dimensional family that generates from the planar vertical-critical periodic orbit $a 1 v$. Specifically, we calculate the 3D families of periodic orbits which generate from the 5 vertical-critical periodic orbits for $\omega=2$, which we already presented in Table 2 .

Three of the families, namely $f a 1 v, f b 1 v$ and $f c 1 v$ consist of plane symmetric periodic orbits, one family $(f c 2 v)$ consists of axisymmetric ones and one family $(f c 3 v)$ consists of doubly symmetric periodic orbits. All the verticalcritical periodic orbits from which the previous mentioned 3D families emanate are unstable. Thus all these 5 families, at least for low inclination, are unstable. Examination of the stability of the five 3D families shows that three of them, namely $f a 1 v, f b 1 v$ and $f c 3 v$, are unstable, while the families $f c 1 v$ for $z \in[0.115,0.173]$ and $f c 2 v$ for $\dot{z} \in[0.482,0.529]$ are stable.

In Fig. 7 we present one three-dimensional periodic orbit for each family and their orthographic projections in the $(x, y)$, $(x, z)$ and $(y, z)$ planes. Underneath each three-dimensional periodic orbit we give the characteristic curves of their family in the $(x, z)$ or $(x, \dot{z})$ and $(x, \dot{y})$ planes. Family $f a 1 v$, as we see from its characteristic curves, is rising in the third dimension and then goes down to the plane to coincide with the second primary body. Families $f c 1 v, f c 2 v$ and $f c 3 v$ also rise in the third dimension and then "terminate" having the second component $\dot{y}$ (or $\dot{y}_{1}$ in the first intersection with $x$-axis) of the velocity vanished and the family changing multiplicity. This fact is apparent and from the corresponding orthographic projection in the $(x, y)$ or $(x, z)$ planes (Figs. $7 \mathrm{e}-7 \mathrm{~g}$ ). Finally, family $f b 1 v$ which is generated from the vertical-critical periodic orbit $b 1 v$, is rising in the third dimension and then returns to the plane again but for $z=3.001$ it collides with the Earth.

\section{Conclusions}

Analytic approximations of periodic motions around the collinear equilibrium points of the restricted three-body problem for varying angular velocity of the primaries, both in plane and in three dimensions, are given. These approximations provide an appropriate tool for the initiation of calculations for the numerical investigation of entire families of large orbits 

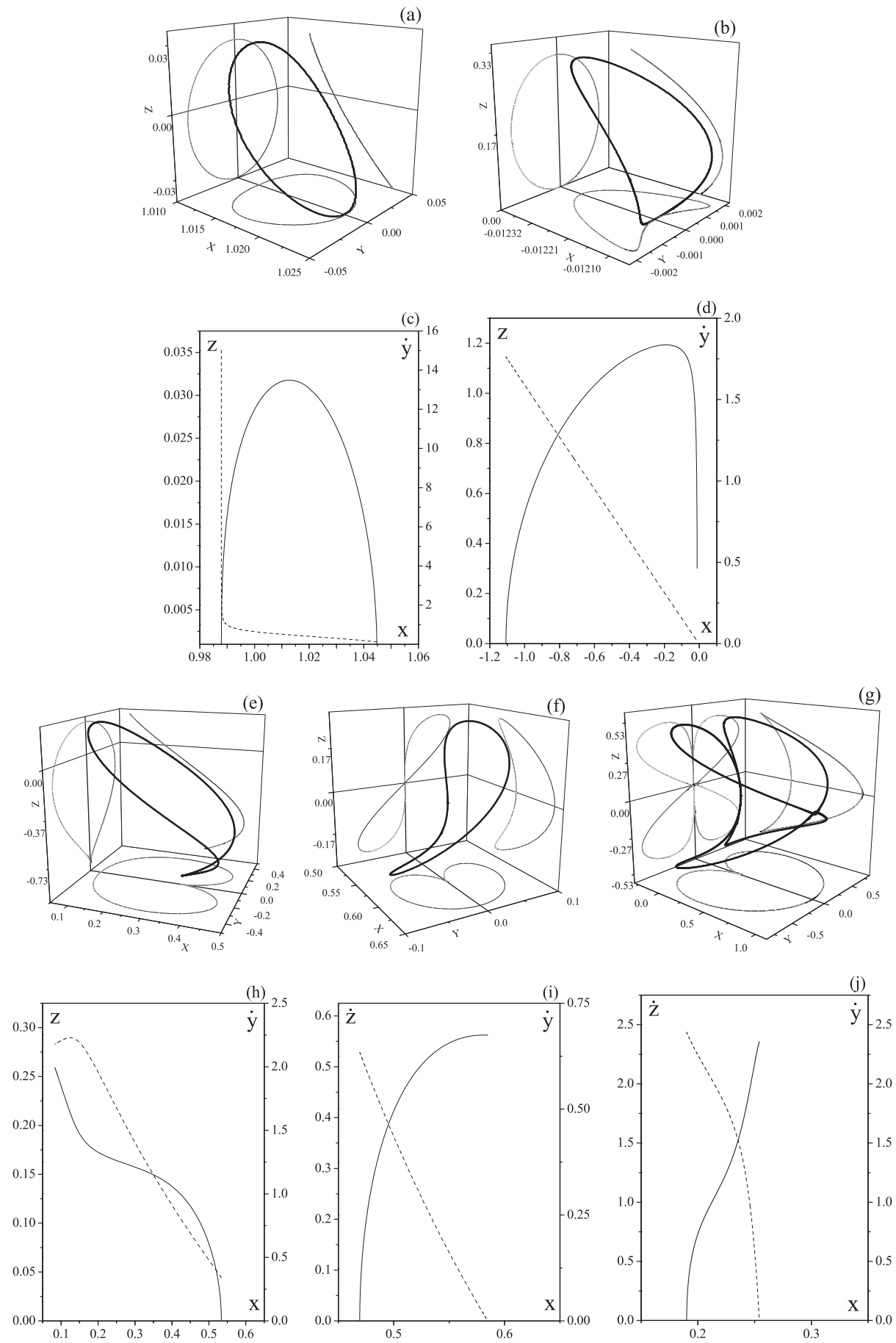

Fig. 7. Three-dimensional periodic orbits with orthographic projections of families $f a 1 v \mathbf{a}), f b 1 v \mathbf{b}), f c 1 v \mathbf{e}), f c 2 v \mathbf{f})$ and $f c 3 v \mathbf{g})$. Frames c), d), h), i), j) The corresponding characteristic curves of previous families in the $(x, z)$ or $(x, \dot{z})$ and $(x, \dot{y})$ planes.

emanating from the corresponding equilibria. So, the families $a, b$ and $c$, which emanate from $L_{2}, L_{3}$ and $L_{1}$ respectively, for the Earth-Moon mass distribution and for $\omega=2$, are calculated and are presented in Fig. 1. It is found that all of them are terminated with collision orbits with one of the primaries. Similarly, we calculated the 3D families which emanate from the collinear equilibrium points and they are presented in Fig. 2. All of them consist of doubly symmetric periodic orbits and the families return to the plane. The terminating orbits are also investigated and found to be vertical-critical orbits of the family $h$ (for the families which emanate from $L_{1}$ and $L_{3}$ ) and of the family $f$ (for the family which emanates from $L_{2}$ ) of the plane problem. This situation is not similar as in the classical restricted three-body problem.

It is found that the angular velocity of the primaries has a strong effect of the families which emanate from the equilibrium configurations, with respect to the classical restricted three-body problem. The evolution of the families as well as their stability which were presented in Fig. 6, is apparent. The number of the vertical-critical periodic orbits changes as the 
angular velocity parameter varies. All the 3D families which emanate from the vertical-critical periodic orbits of families $a, b$ and $c$, for $\mu=0.01213$ and $\omega=2$, are calculated and their characteristic curves as well as one member of each 3D families, are illustrated in Fig. 7.

Summarizing the results of the three-dimensional families, for $\omega=2$ and $\mu=0.01213$, we conclude the following: From each collinear equilibrium point $L_{1}$ and $L_{3}$ emanates one 3D family of doubly symmetric periodic orbits which terminates on the plane again, at a vertical-critical planar symmetric periodic orbit of family $h$. Similarly, from $L_{2}$ starts one 3D family which terminates at a vertical-critical plane symmetric periodic orbit of family $f$. Three families of planar symmetric periodic orbits of which the first, namely $f a 1 v$, starts at the vertical-critical planar periodic orbit $a 1 v$ of family $a$ and at the end coalesces with the Moon. The second one, namely $f b 1 v$, starts at the vertical-critical planar periodic orbit $b 1 v$ of family $b$ and terminates on the Earth, and the third, namely $f c 1 v$, starts at the verticalcritical planar periodic orbit $c 1 v$ of family $c$ and rises in the third dimension and then changes multiplicity. A 3D family of axisymmetric periodic orbits, namely $f c 2 v$, starts at the vertical-critical planar periodic orbit $c 2 v$ of family $c$ and terminates after changing its multiplicity. Finally, a $3 \mathrm{D}$ family of doubly symmetric periodic orbits, namely $f c 3 v$, starts at the vertical-critical planar periodic orbit $c 3 v$ of family $c$ and terminate again after changing its multiplicity.

\section{References}

Chermnykh, S. V. 1987, Vest. Leningrad Univ., 2(8), 10.

Goździewski, K., \& Maciejewski, A. J. 1998, Celest. Mech. Dynam., 70,41

Hénon, M. 1965, Ann. Astrophys., 28, 992

Hénon, M. 1969, A\&A, 1, 223

Hénon, M. 1973, A\&A, 28, 415

Hénon, M., \& Guyot, M. 1970, in Periodic Orbits, Stability and Resonances, ed. G. E. O. Giacaglia (Dordrecht-Holland: Reidel), 349

Marchal, C. 1990, The three body problem (Amsterdam: Elsevier Science Publishers B.V.)

Markellos, V. V., Goudas, C. L., \& Katsiaris, G. A. 1981, in Investigating the Universe (Dordrecht, Holland: D. Reidel Publ. Co.)

Papadakis, K. E., Perdios, E. A., \& Markellos, V. V. 1988, Ap\&SS, 140,373

Perdios, E. A., \& Ragos, O. 2004, A\&A, 414, 361

Szebehely, V. G. 1967, Theory of Orbits (London and New York: Academic Press)

Zagouras, C., \& Markellos, V. V. 1985, Celest. Mech., 35, 257 